\title{
Point Defects in Turbostratic Stacked Bilayer Graphene
}

\author{
Chuncheng Gong ${ }^{1}$, Gun-Do Lee ${ }^{2}$, Jamie H. Warner ${ }^{1}$ \\ ${ }^{1}$ Department of Materials, University of Oxford, Parks Road, Oxford, OX1 3PH, United Kingdom \\ ${ }^{2}$ Department of Materials Science and Engineering, Seoul National University, Seoul, 151-742, Korea
}

\begin{abstract}
KEYWORDS: point defects, monovacancy, divacancy, bond rotation
\end{abstract}

The electronic, mechanical and magnetic properties of graphene can be greatly impacted by defects within the lattice. ${ }^{1-6}$ Thus, it is of significant importance to understand the structure and behavior of these defects in order to realize the multiple applications of graphene, such as electronic devices. ${ }^{7,8}$ Monovacancy (MV) and divacancy (DV) are the simplest form of vacancies in graphene, which have been investigated by various experimental and theoretic researches. ${ }^{9-17}$ Figure 1a-c schematically illustrates the formation process of a monovacancy defect. The carbon atom colored in green is first removed from the pristine graphene lattice, which leaves three dangling bonds as shown in Figure 1b. First principle calculations indicate that two of the three under-coordinated carbon atom can further go through a bond reconstruction named Jahn-Teller distortion, yielding the formation of a closed 5- and a 9- membered rings (Figure 1c). ${ }^{9-}$ ${ }^{14}$ El-Barbary et al. has demonstrated that Jahn-Teller distortion gives a $\sim 0.2 \mathrm{eV}$ lower energy. ${ }^{14}$ According to their calculation, the remaining under-coordinated atom is moved out of the plane by $\Delta z \sim$ $0.5 \AA$ due to the increase in the local density of states at the dangling bond. Robertson et al. has characterized MVs in monolayer graphene using an aberration-corrected HR-TEM equipped with a monochromated beam source and have demonstrated that Jahn-Teller reconstructed MV gives rise to extra strain compared to the symmetric one. ${ }^{15}$ Theoretical studies have suggested that the migration of 
MVs takes place by switching one of the edge atoms to the opposite site and thus shifting the vacancy to a neighboring position, with an activation barrier of only $\sim 1.5 \mathrm{eV} \cdot{ }^{14,16}$

The divacancy (DV) defect is the most frequently observed vacancy structure in the AC-TEM inspection of graphene. It can be created by removing a pair of neighboring atoms from graphene lattice or by the combination of two MVs. Figure 1d shows a pentagon-octagon-pentagon [DV 1 (5-8-5)] sequence with no dangling bond after the removal of two carbon atoms from graphene lattice. Nevertheless, it is not the only configuration of a DV. Figure $1 \mathrm{e}$ and $\mathrm{f}$ show that it could reconstruct into two more complex structures $\left[\mathrm{DV}_{2}(555-777)\right.$ and $\left.\mathrm{DV}_{3}(5555-6-7777)\right]$ via a series of $\mathrm{SW}$ bond rotations. The detailed transformation process will be discussed in Figure 5. The formation energy of all three DVs is around 8 $\mathrm{eV}$ given by DFT calculation, which is in the same magnitude as that of MV $(7.5 \mathrm{eV}){ }^{14,16}$ This is understandable as the minimum energy required to sputter the under-coordinated carbon atom in a MV is $\sim 14 \mathrm{eV}$, significantly lower than the sputtering threshold for pristine graphene lattice $(\sim 22 \mathrm{eV}) .{ }^{18,19}$ Thus one would expect a MV to quickly convert into a DV under electron beam irradiation. The migration barrier for a DV is $\sim 7 \mathrm{eV}$ which is also higher than that of a $\mathrm{MV}(\sim 1.5 \mathrm{eV})$ making DVs highly immobile at room temperature. ${ }^{14}$

Despite the point defects in graphene have been well studied in monolayer graphene, the direct imaging of defects in graphene with $\geq 2$ layers is limited. This is primarily because that the contrast from different layers overlaps, making it difficult to interpret specific defect structures. In this work, we identify the atomic structure and dynamics of MVs and DVs in turbostratic stacked bilayer graphene. By applying different negative masks in FFTs, we managed to separate the contrast of the two layers and characterize the defect in either layer. We find that the stability and migration behavior of MV and DV in bilayer graphene is apparently different from those in monolayer. To be confirmed by the TBMD and DFT calculation results. Bilayer graphene was synthesized by ambient pressure chemical vapor deposition method (CVD) performed on a molten copper substrate as previously reported, ${ }^{20}$ before being transferred onto a holy $\mathrm{S}_{\mathrm{i} 3} \mathrm{~N}_{4}$ grid for AC-TEM imaging using Oxford's JEOL 2200MCO AC-TEM equipped with a 

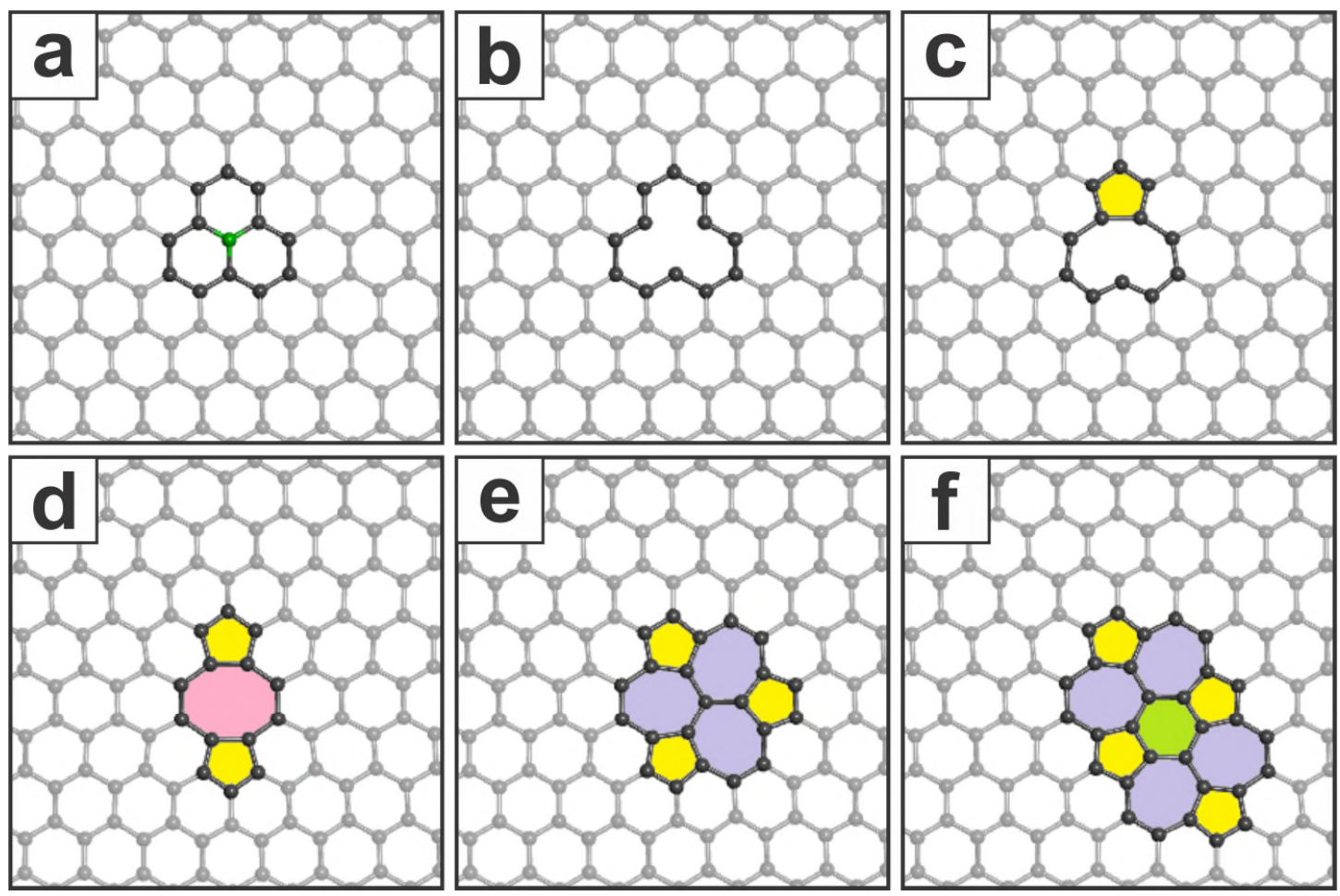

Figure 1. Atomic models showing the formation of monovacancy (MV) and the three different configurations of divacancies (DVs) in graphene. (a) The carbon atom highlighted in green is removed from the pristine graphene lattice, yielding the formation of the vacancy structure shown in (b). (c) The structure in (b) undergoes a Jahn-Teller distortion and generates a closed 5- and 9-membered ring. (d-e) Atomic models of (d) $\mathrm{DV}_{1}$ (5-8-5), (e) $\mathrm{DV}_{2}$ (555-777) and (f) $\mathrm{DV}_{3}$ (5555-6-7777). The color scheme in the atomic models represents the number of carbon atoms in each ring, with $5=$ yellow, 6 (rotated) $=$ green, $7=$ blue and $8=$ pink.

\section{Results and Discussion}



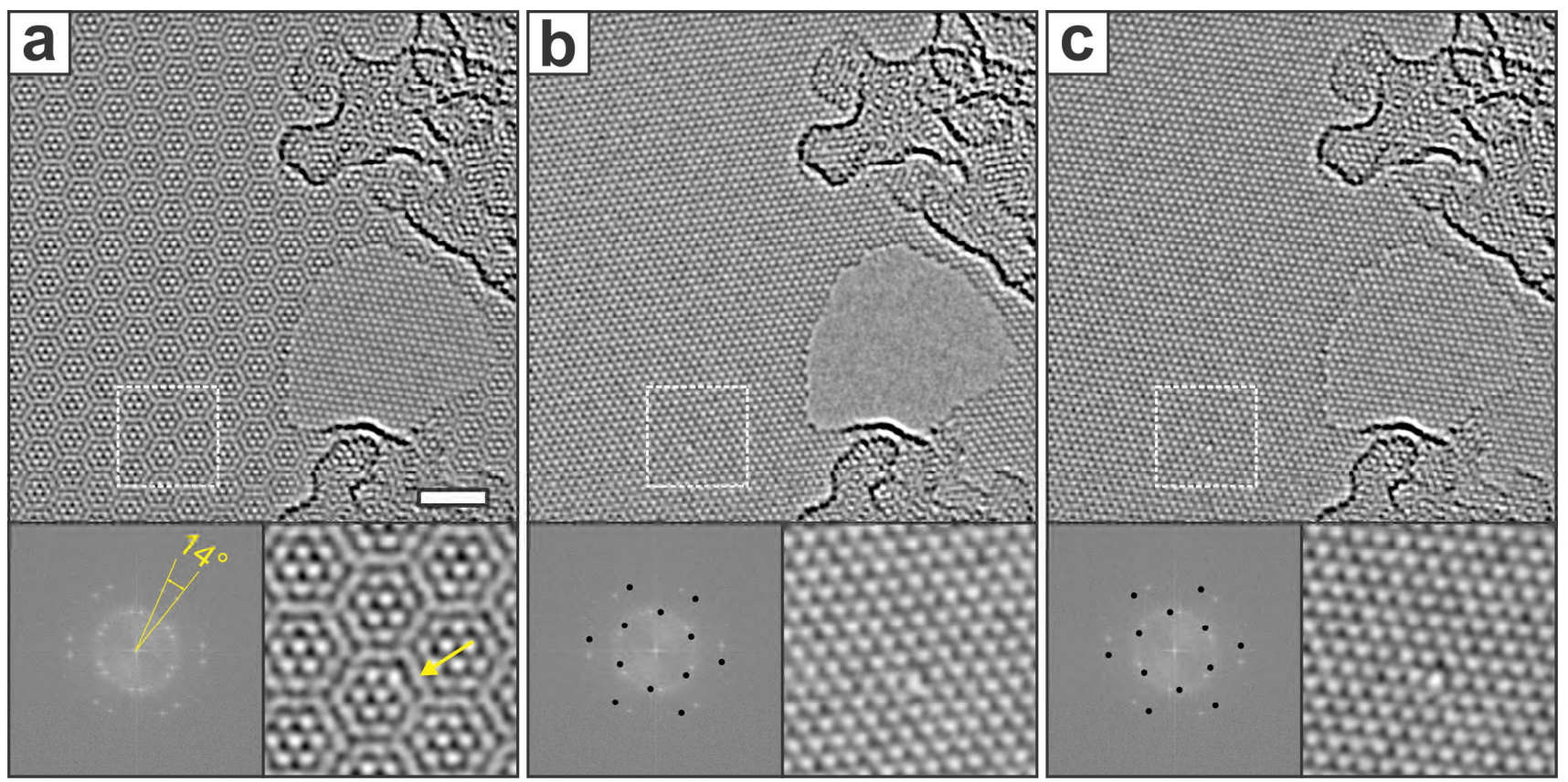

Figure 2. AC-TEM image of two turbostratic stacked graphene layers. (a) Smoothed AC-TEM images of the bilayer graphene with its FFT and magnified view of the area cropped by the white dashed square shown in the bottom insets. The yellow arrow indicates the lattice irregularity in Moiré pattern. $(b, c)$ The two layers are separated by placing a negative mask on one of the two sets of hexagonal patterns in the FFT. The scale bar in panel a is $2 \mathrm{~nm}$.

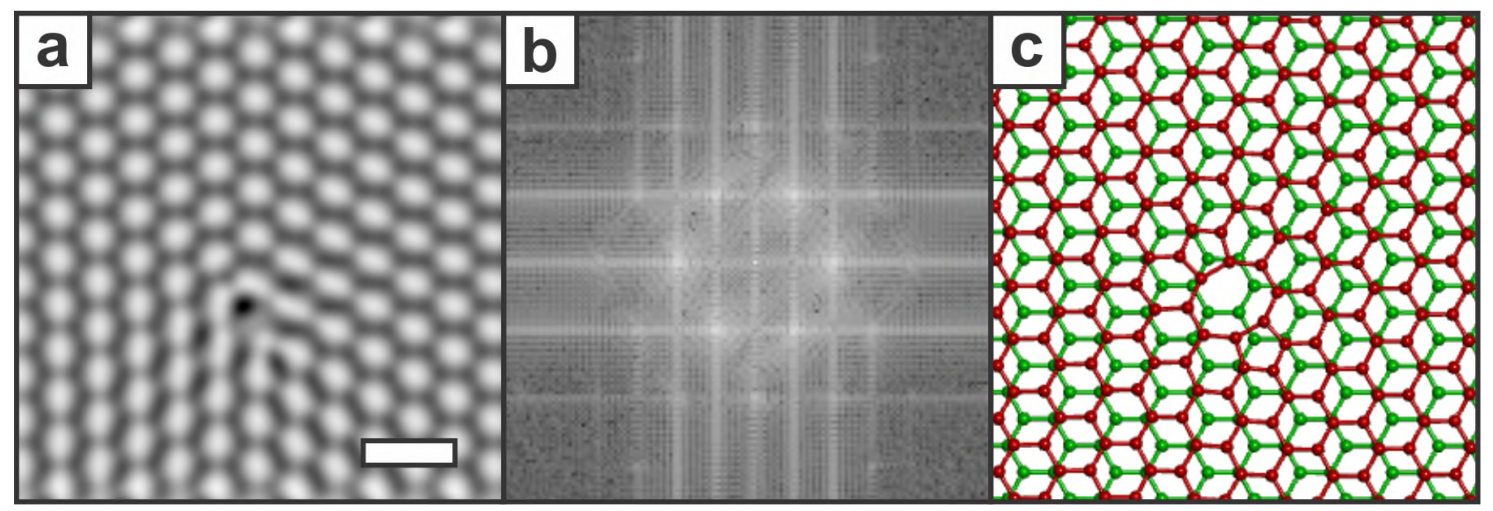

Figure 3. 5-8-5 $\mathrm{DV}_{1}$ in $\mathbf{A B}$ stacked bilayer graphene. (a) Multislice simulated TEM image using the atomic model shown in (c). (b) FFT of (a). The scale bar in panel a is $0.5 \mathrm{~nm}$.

In Figure 2a, a smoothed AC-TEM image of the turbostratic stacked bilayer graphene is shown. Its FFT (bottom left inset) shows two sets of hexagonal patterns and suggests that the mismatch angle between the two layers is $14^{\circ}$. Examination of the area cropped by the white square (bottom right inset) indicates visible lattice irregularity in Moiré pattern, highlighted by the yellow arrow, which could result from the 
defect in one or both of the two layers. By placing a negative mask on one of the two hexagonal patterns, we managed to separate the individual contrast of the two twisted layers. By looking into the atomic structure of either layer, the presence of a MV is revealed which locates in the same layer with the hole. However, as the negative mask in the FFT only removes the periodic structure, the defect-free graphene layer is still overlaid with the contrast from the MV, edge of the hole and amorphous carbon adsorbates after FFT reconstruction. As a result, the hexagons near the position of MV is a bit distorted and the edge of the hole and amorphous contamination still exist in Figure 2c. Figure S1 shows the AC-TEM image of a 555-777 $\mathrm{DV}_{2}$ which is processed the same way. The defect-free layer was also overlapped with the contrast from the $\mathrm{DV}_{2}$ defect. (In Figure $S 2$ different positive masks are applied to identify of the DV exists in only one graphene layer or is an interlayer defect. However, the reconstructed image shows no MV or DV in their original places, only perfect graphene lattice.) However, such method can be only applied in twisted bilayer graphene. In Figure 3, a multislice simulated TEM image of a 5-8-5 DV 1 in $A B$ stacked bilayer graphene is shown, in which the defect is overlaid with the signal of the other graphene layer, which prohibited us from resolving detailed atomic structures. Nevertheless, the two graphene layers correspond to the same set of hexagonal spots in the FFT (Figure 3b) and cannot be separated using the approach shown in Figure 2. 


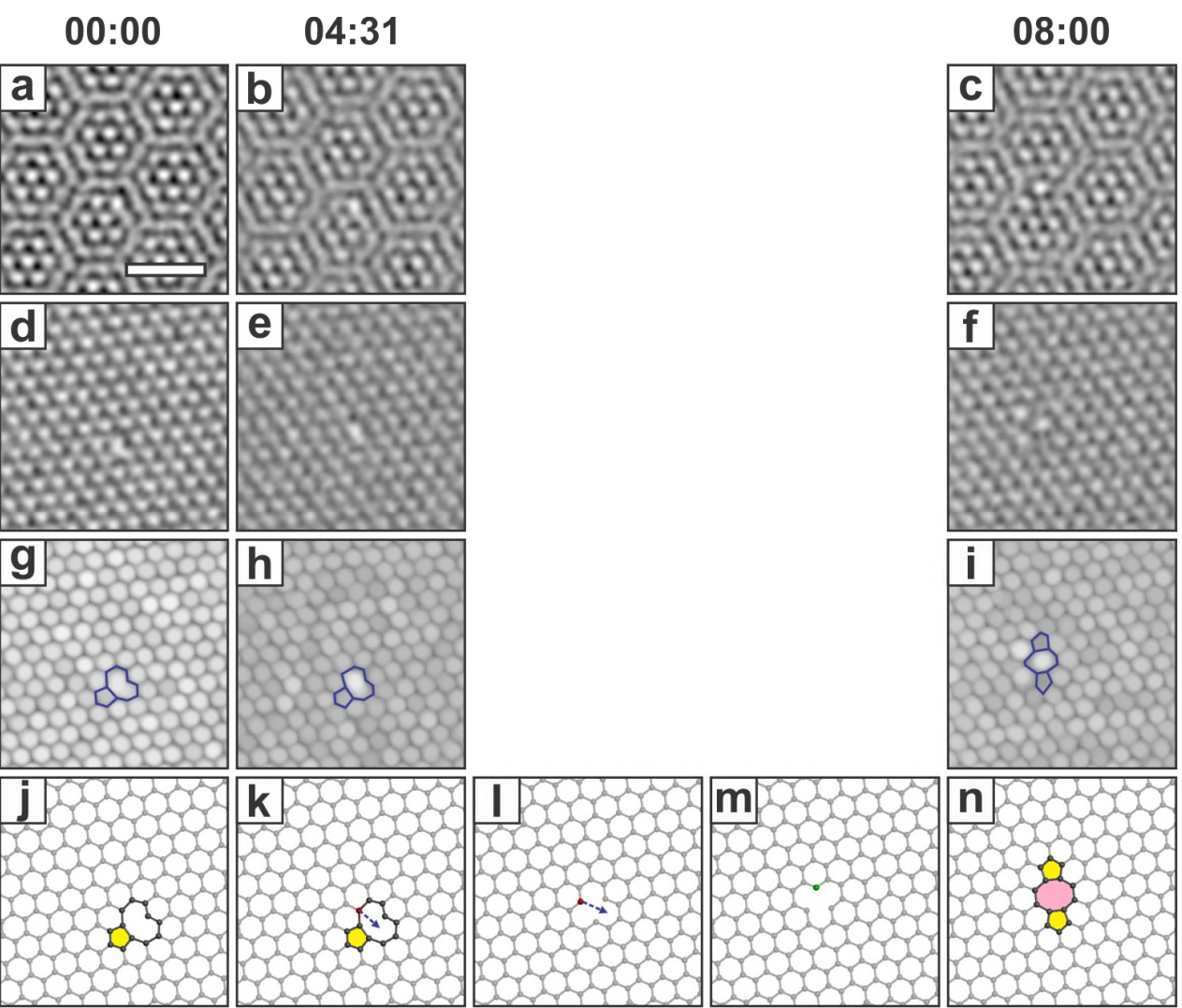

Figure 4. The migration of a monovacancy which subsequently transformed into a 5-8-5 DV in bilayer graphene. $(\mathrm{a}-\mathrm{c})$ Smoothed time series AC-TEM images of the cropped area shown in Figure 1. $(\mathrm{d}-\mathrm{f})$ Corresponding reconstructed images from the negative mask shown in the FFT in Figure 2b. (g-i) Maximum filtered images of $(\mathrm{d}-\mathrm{f})$ with non-hexagonal rings highlighted by blue polygons. $(\mathrm{j}, \mathrm{k}, \mathrm{n})$ Atomic models of $(\mathrm{d}-\mathrm{f}) .(\mathrm{k}-\mathrm{n})$ Atomic models showing a possible pathway of the step-by-step structural reconfiguration between panel $\mathrm{k}$ to $\mathrm{n}$. The atoms to migrate are highlighted in red with the migration direction indicated by the blue dashed arrows. Atoms to be sputtered are colored in green. The color scheme in the atomic models represents the number of carbon atoms in each ring, with $5=$ yellow and 8 $=$ pink. The scale bar in panel a is $1 \mathrm{~nm}$.

Our data set in total consists of 66 AC-TEM frames taken over a period of 16 min $15 \mathrm{~s}$. The time interval between two consecutive frames is about $15 \mathrm{~s}$. Figure 4a,d show the same MV in Figure 2. Despite the energy barrier for a MV to migrate is only $\sim 1.5 \mathrm{eV}$ in single layer graphene, ${ }^{14,16}$ the defect in Figure $4 \mathrm{a}$ remains unchanged for more than $4 \min 31 \mathrm{~s}$, before losing the under-coordinated carbon atom and generating a 5-8-5 divacancy. May need some calculation to confirm the stability of MV in bilayer graphene. This is probably due to that the migration barriers for $M V$ and $D V$ defects in bilayer graphene 
is different from those in monolayer graphene, as the under-coordinated carbon atom may bond with the defect-free layer. Figure $4 \mathrm{k}-\mathrm{n}$ suggest a plausible path way for the structural transformation from panel a to $\mathrm{c}$, in which the monovacancy first migrated via the diffusion of the under-coordinated atom (colored in red in Figure 4k,1). The migration directions are highlighted by the blue dashed arrows. Afterward the dangling bond highlighted in green was sputtered under electron irradiation, yielding a 5-8-5 DV (Figure 4c,f). There are a few frames between Figure $2 b$ and c but lack sufficient spatial resolution to resolve the atomic structure of defects.

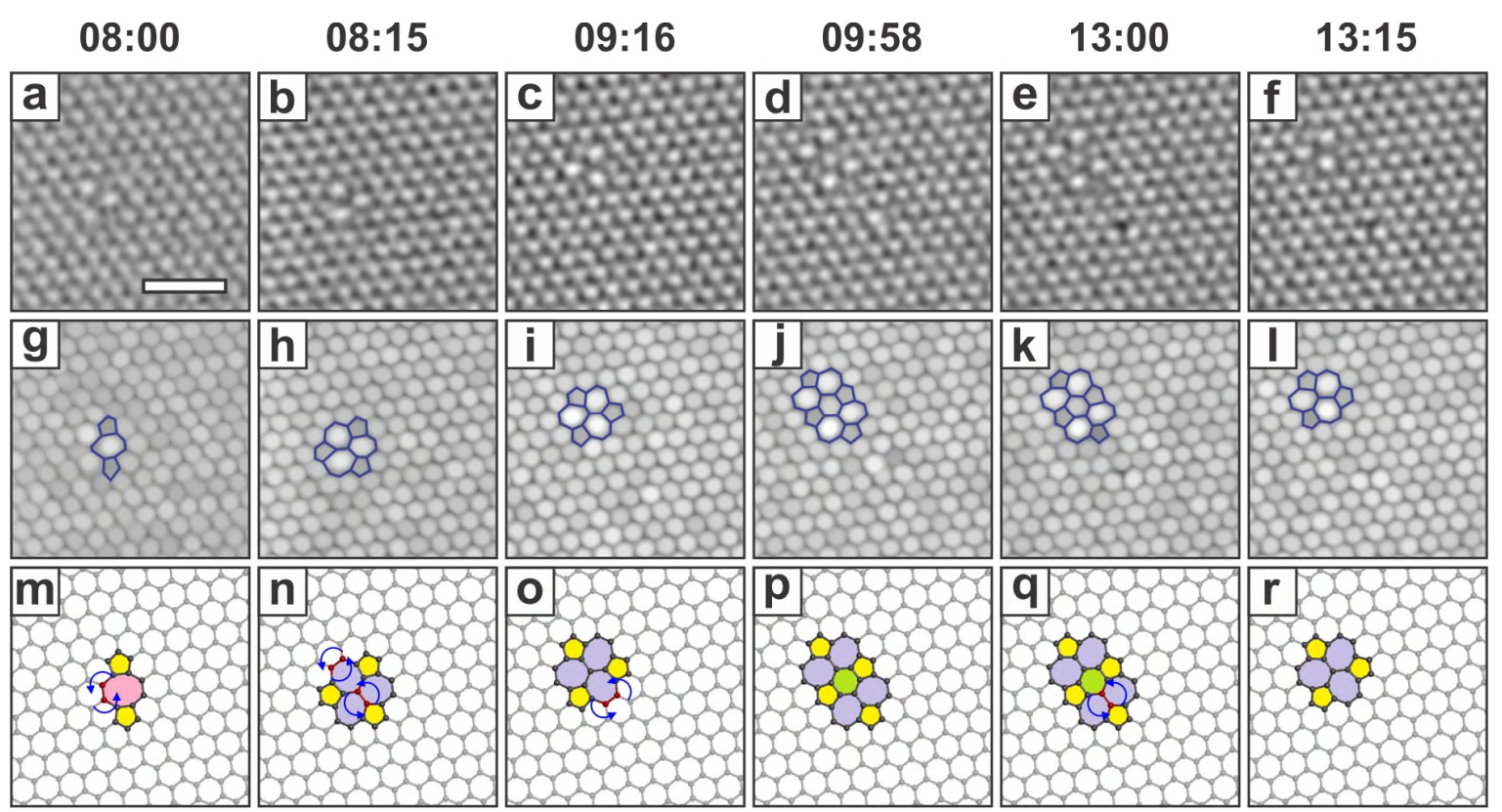

Figure 5. The migration and reconfiguration of a divacancy in bilayer graphene. (a-f) Time sequential FFT reconstructed images. (g-i) Maximum filtered images of (a-f) with non-hexagonal rings highlighted by blue polygons. $(\mathrm{m}-\mathrm{r})$ Atomic models of $(\mathrm{a}-\mathrm{f})$ with the atoms to rotate highlighted in red and by arrows. The color scheme in the atomic models represents the number of carbon atoms in each ring, with $5=$ yellow, 6 (rotated) $=$ green, $7=$ blue and $8=$ pink. The scale bar in panel a is $1 \mathrm{~nm}$.

Previous research has suggested that the random walk of a DV in monolayer graphene can be achieved by switching between configurations, such as $\mathrm{DV}_{1}(5-8-5) \rightarrow \mathrm{DV}_{2}(555-777) \rightarrow \mathrm{DV}_{1}(5-8-5){ }^{21}$ In this work, we observed similar phenomena in bilayer graphene. Figure 5 shows how bond rotations switch the configuration of the DV and drive it to migrate. In Figure 3a, the rotation of a carbon dimer in the octagon first transformed the 5-8-5 $\mathrm{DV}_{1}$ into the 555-777 $\mathrm{DV}_{2}$. Then the $\mathrm{DV}_{2}$ migrated via two bond rotations 
highlighted by blue arrows in Figure 3n. After the transformation the $\mathrm{DV}_{2}$ remained its atomic configuration but rotated by $30^{\circ}$. The defect was switched to the 5555-6-7777 configuration and remained stable for about 3 min before revering back via a further bond rotation in $15 \mathrm{~s}$.

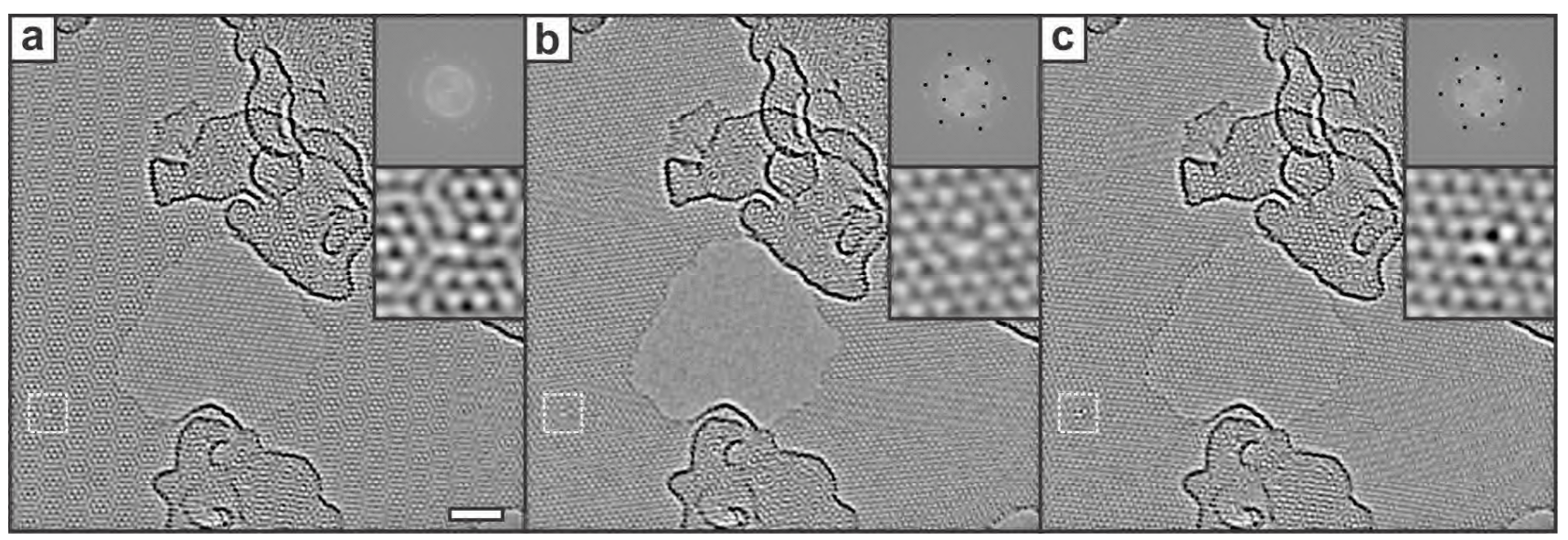

Figure S1. AC-TEM image of a 555-777 $\mathrm{DV}_{2}$ in turbostratic stacked bilayer graphene. (a) AC-TEM image of the bilayer graphene with its FFT and magnified view of the area cropped by the white dashed square shown in the insets. $(b, c)$ The two layers are separated by placing a negative mask on one of the two sets of hexagonal patterns in the FFT. The scale bar in panel a is $2 \mathrm{~nm}$. 


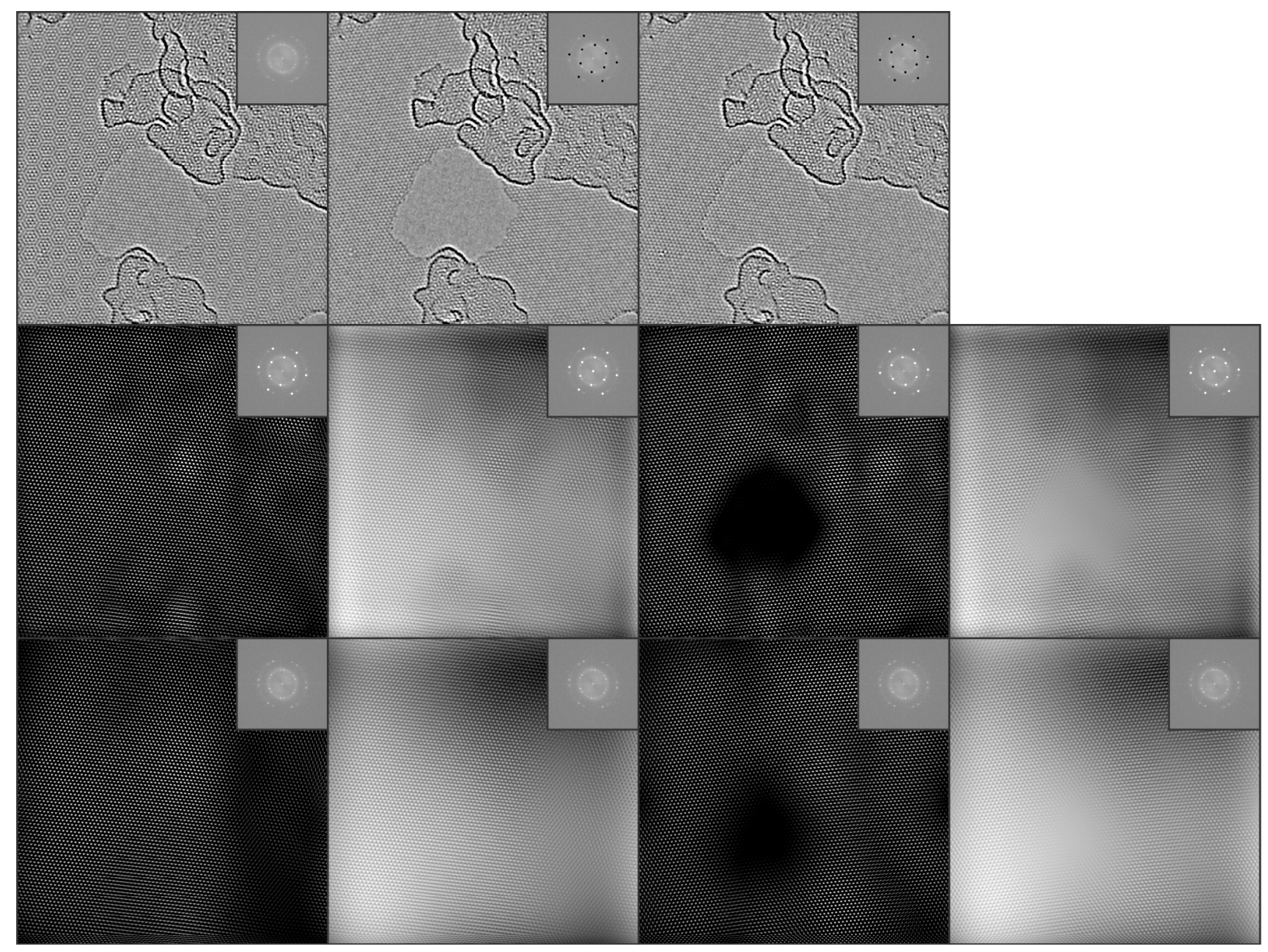

Figure S2. AC-TEM image of a 555-777 $\mathrm{DV}_{2}$ in turbostratic stacked bilayer graphene with white masks applied in the FFT.

\section{Methods}

\section{Synthesis of graphene}

Graphene was synthesized by atmospheric pressure chemical vapor deposition (CVD) method using a melted copper sheet as the catalytic as previously reported ${ }^{20}$ The high purity copper foil (Alfa Aesar, Puratonic $99.999 \%$ pure, $0.1 \mathrm{~mm}$ thick) of $\sim 1 \mathrm{~cm}^{2}$ were placed on the molybdenum piece of same size(Alfa Aesar, $99.95 \%$ pure, $0.1 \mathrm{~mm}$ thick), which were both loaded into a 1 inch quartz tube in the CVD system. Molybdenum act as a stable wetting layer to prevent liquid copper from balling. 100 s.c.c.m. $\mathrm{H}_{2} / \mathrm{Ar}\left(20 \% \mathrm{H}_{2}\right.$ in $\left.\mathrm{Ar}\right), 100$ s.c.c.m. $\mathrm{CH}_{4}\left(1 \% \mathrm{CH}_{4}\right.$ in $\left.\mathrm{Ar}\right)$ and 200 s.c.c.m. $100 \%$ Ar were flowed for 30 
minutes. $\mathrm{CH}_{4}$ flow was switched off before increasing hot-zone temperature to $1090{ }^{\circ} \mathrm{C}$. Once the temperature reached $1090^{\circ} \mathrm{C}$, the quartz tube was moved from the room temperature zone to the center of heating zone and annealed for 30 minutes. The flow of $\mathrm{H}_{2} / \mathrm{Ar}$ was then reduced to 80 s.c.c.m. and 10 s.c.c.m. of $1 \% \mathrm{CH}_{4}$ in $\mathrm{Ar}$ was added for $90 \mathrm{~min}$ for graphene growth. After growth, the quartz was removed from the heating zone for rapid cooling in the air with $\mathrm{CH}_{4}$ off.

\section{Transfer}

A PMMA scaffold (8\% wt in anisole, $495 \mathrm{k}$ molecular weight) was spin-coated onto the graphene sheet at $4700 \mathrm{rpm}$ for $60 \mathrm{~s}$ and then baked at $180^{\circ} \mathrm{C}$ for $90 \mathrm{~s}$ to solidify. Afterwards the sample was made up of a molybdenum/copper/graphene/PMMA stack. The copper layer were etched by floating the sample on the mixed solution of iron (III) chloride and hydrochloride, leaving a floating graphene-PMMA film on the top after $48 \mathrm{~h}$. The film was collected using a clean glass slide and transferred onto the surface of the DI water for 30 min wash away remaining iron (III) chloride. To further dissolve excess iron chloride, the sample was transferred onto a $10 \%$ hydrogen chloride solution for $5 \mathrm{~min}$, before rinsed again in the DI water for 30 min. A holey $\mathrm{Si}_{3} \mathrm{~N}_{4}$ TEM grid (Agar Scientific Y5358) was mounted onto an adhesive silicon chip and was used to scoop up the floating graphene/PMMA film. Afterward, the grid on the silicon chip was moved onto a glass slide and dried in the air for $24 \mathrm{~h}$ and subsequently baked at $150{ }^{\circ} \mathrm{C}$ to relax for 15 min. The grid on the silicon chip was then cured at $350{ }^{\circ} \mathrm{C}$ for $12 \mathrm{~h}$ to burn out PMMA, leaving only graphene on the holey $\mathrm{Si}_{3} \mathrm{~N}_{4}$ grid.

\section{Electron Microscopy}

AC-TEM images were taken at an accelerating voltage of $80 \mathrm{kV}$, using Oxford's JEOL JEM-2200MCO field emission transmission electron microscope with a CEOS image corrector. ${ }^{22}$ Defects were introduced using a focused electron beam according to our previously reported procedure. ${ }^{23}$ A double Wien filter monochromator with a $7 \mu \mathrm{m}$ slit was used to reduce the energy spread of the electron beam to $\sim 0.21 \mathrm{eV}$. Data were recorded using a Gatan Ultrascan $4 \mathrm{~K} \times 4 \mathrm{~K}$ CCD camera with $2 \mathrm{~s}$ acquisition times and 2 pixel 
binning. TEM images were processed using Image J. Smoothing of images was achieved by using a Gaussian blur filter in Image J.

\section{In situ Heating Holder}

To perform variable-temperature experiments, we used a commercially available in situ heating holder from DENS Solutions (SH30-4M-FS). In the DENS Solutions holder, heating the sample was achieved by passing a current through a platinum resistive coil imbedded in the TEM chip (DENS Solutions DENSC-30). The resistance of the platinum coil is monitored in a four-point configuration, and the temperature is calculated using the Callendar-Van Dusen equation (with calibration constants provided by the manufacturer).

\section{Acknowledgment}

J.H.W. thanks the support from the Royal Society. C.G. thanks the support form Clarendon Fund Scholarship.

\section{Reference}

(1) Castro Neto, a. H.; Peres, N. M. R.; Novoselov, K. S.; Geim, A. K. The Electronic Properties of Graphene. Rev. Mod. Phys. 2009, 81, 109-162.

(2) Shi, H.; Barnard, A. S.; Snook, I. K. Site-Dependent Stability and Electronic Structure of Single Vacancy Point Defects in Hexagonal Graphene Nano-Flakes. Phys. Chem. Chem. Phys. 2013, 15, 4897-4905.

(3) Lee, C.; Wei, X.; Kysar, J. W.; Hone, J. Measurement of the Elastic Properties and Intrinsic Strength of Monolayer Graphene. Science 2008, 321, 385-388.

(4) Yazyev, O. Magnetism in Disordered Graphene and Irradiated Graphite. Phys. Rev. Lett. 2008, $101,037203$.

(5) Wang, B.; Pantelides, S. T. Magnetic Moment of a Single Vacancy in Graphene and Semiconducting Nanoribbons. Phys. Rev. B: Condens. Matter Mater. Phys. 2012, 86.

(6) Nair, R. R.; Sepioni, M.; Tsai, I.-L.; Lehtinen, O.; Keinonen, J.; Krasheninnikov, a. V.; Thomson, T.; Geim, a. K.; Grigorieva, I. V. Spin-Half Paramagnetism in Graphene Induced by Point Defects. Nat. Phys. 2012, 8, 199-202.

(7) Novoselov, K. S.; Fal'ko, V. I.; Colombo, L.; Gellert, P. R.; Schwab, M. G.; Kim, K. A Roadmap 
for Graphene. Nature 2012, 490, 192-200.

(8) Schwierz, F. Graphene Transistors. Nat. Nanotechnol. 2010, 5, 487-496.

(9) Lu, A.; Pan, B. Nature of Single Vacancy in Achiral Carbon Nanotubes. Phys. Rev. Lett. 2004, 92 , 105504.

(10) Rossato, J.; Baierle, R. J.; Fazzio, A.; Mota, R. Vacancy Formation Process in Carbon Nanotubes: First-Principles Approach. Nano Lett. 2005, 5, 197-200.

(11) Berber, S.; Oshiyama, A. Reconstruction of Mono-Vacancies in Carbon Nanotubes: Atomic Relaxation vs. Spin Polarization. Phys. B Condens. Matter 2006, 376, 272-275.

(12) Lehtinen, P.; Foster, A.; Ma, Y.; Krasheninnikov, A.; Nieminen, R. Irradiation-Induced Magnetism in Graphite: A Density Functional Study. Phys. Rev. Lett. 2004, 93, 187202.

(13) Ewels, C.; Telling, R.; El-Barbary, A.; Heggie, M.; Briddon, P. Metastable Frenkel Pair Defect in Graphite: Source of Wigner Energy? Phys. Rev. Lett. 2003, 91, 025505.

(14) El-Barbary, A.; Telling, R.; Ewels, C.; Heggie, M.; Briddon, P. Structure and Energetics of the Vacancy in Graphite. Phys. Rev. B 2003, 68, 144107.

(15) Robertson, A. W.; Montanari, B.; He, K.; Allen, C. S.; Wu, Y. A.; Harrison, N. M.; Kirkland, A. I.; Warner, J. H. Structural Reconstruction of the Graphene Monovacancy. ACS Nano 2013, 7, $4495-4502$.

(16) Krasheninnikov, A. V.; Lehtinen, P. O.; Foster, A. S.; Nieminen, R. M. Bending the Rules: Contrasting Vacancy Energetics and Migration in Graphite and Carbon Nanotubes. Chem. Phys. Lett. 2006, 418, 132-136.

(17) Chen, Q.; Robertson, A. W.; He, K.; Gong, C.; Yoon, E.; Lee, G.-D.; Warner, J. H. Atomic Level Distributed Strain within Graphene Divacancies from Bond Rotations. ACS Nano 2015, 9, 85998608.

(18) Smith, B. W.; Luzzi, D. E. Electron Irradiation Effects in Single Wall Carbon Nanotubes. J. Appl. Phys. 2001, 90, 3509-3515.

(19) Banhart, F. Irradiation Effects in Carbon Nanostructures. Reports Prog. Phys. 1999, 62, 11811221.

(20) Wu, Y. A.; Fan, Y.; Speller, S.; Creeth, G. L.; Sadowski, J. T.; He, K.; Robertson, A. W.; Allen, C. S.; Warner, J. H. Large Single Crystals of Graphene on Melted Copper Using Chemical Vapor Deposition. ACS Nano 2012, 6, 5010-5017.

(21) Kotakoski, J.; Mangler, C.; Meyer, J. C. Imaging Atomic-Level Random Walk of a Point Defect in Graphene. Nat. Commun. 2014, 5, 3991.

(22) Warner, J. H.; Margine, E. R.; Mukai, M.; Robertson, A. W.; Giustino, F.; Kirkland, A. I. Dislocation-Driven Deformations in Graphene. Science 2012, 337, 209-212.

(23) Robertson, A. W.; Allen, C. S.; Wu, Y. A.; He, K.; Olivier, J.; Neethling, J.; Kirkland, A. I.; Warner, J. H. Spatial Control of Defect Creation in Graphene at the Nanoscale. Nat. Commun. 2012, 3, 1144 . 